\section{Brazil launches an innovative program to develop the National Institutes for Science and Technology (INCTs): the INCT for Translational Medicine}

\section{Brasil lança um progirama inovador para desenvolver os Institutos Nacionais de Ciência e Tecnologia (INCTs): o INCT para Medicina Translacional}

The Brazilian System of Science and Technology has launched the National Institutes for Science and Technology which are intended as nation-wide networks of research. This initiative has been coordinated by the Ministry of Science and Technology and the Conselho Nacional de Desenvolvimento Científico e Tecnológico (CNPq); the Institutes were officially launched in November 2008. The budget for this initiative is about US $\$ 260$ million over the next five years. The Institutes will occupy a strategic position in the country and will be run in partnership with six State-run foundations for research support, in a joint effort with the Ministry of Health and Education, Coordenação de Aperfeiçoamento de Pessoal de Nivel Superior (CAPES), Petrobras, and the National Bank for Social and Economic Development (BNDES).

The INCT for Translational Medicine represents perhaps a unique opportunity in the field of health-related research, since it offers a potential means of applying the findings obtained in basic research to everyday healthcare. The group awarded with the INCT-TM has become accustomed to the concept of translational research in its efforts to apply the advances made in the field of growth factors research to applications in psychiatry and neurology. In fact, the free enterprise sector has been faster than the Academia in using translational research to boost the development of products and patents. In this particular field, the Brazilian structure for research development is rather poor: while $2 \%$ of the science produced in the world comes from Brazil, only $0.1 \%$ of the total patents are filed by Brazilian researchers, a 20-fold gap. The INCT-TM has been designed to fill this gap within the area of health-related research. It was conceived as the collaborative effort of six centers: UFRGS, USP-RP, UFRJ, PUC-RS, UNESC and a core facility in which over 70 researchers and 150 students participate directly. The INCT-TM also has a wide interaction with several Brazilian and international research groups from different countries such as Canada, the UK, the USA and Spain.

Thus, we expect that the results of the ongoing and future studies of the INCT-TM will help Brazilian science to develop a translational edge, with greater attention paid to the potential benefits of using new findings from basic science as a means to achieve more effective healthcare.

Flávio Kapczinski National Science and Technology Institute (INCT) for

Translational Medicine Molecular Psychiatry Laboratory, Centro de Pesquisas, Hospital de Clínicas de Porto Alegre (HCPA), Universidade Federal do Rio Grande do Sul (UFRGS), Porto Alegre (RS), Brazil 
Jaime Eduardo Hallak

National Science and Technology Institute (INCT) for

Translational Medicine

Neurosciences and Behavior Department, Faculdade de Medicina de Ribeirão Preto (FMRP),

Universidade de São Paulo (USP), Ribeirão Preto (SP), Brazil

\section{Antonio Egidio Nardi}

National Science and Technology Institute (INCT) for

Translational Medicine

Laboratory of Panic and Respiration, Institute of Psychiatry,

Universidade Federal do Rio de Janeiro (UFRJ), Rio de Janeiro (RJ), Brazil

\section{Rafael Roesler}

National Science and Technology Institute (INCT) for Translational Medicine Laboratory of Molecular Neuropharmachology, Department of Pharmacology, Instituto de Ciências Básicas da Saúde, Universidade Federal do Rio Grande do Sul (UFRGS), Porto Alegre (RS), Brazil Cancer Research Laboratory, Experimental Research Center, Hospital de Clínicas de Porto Alegre (HCPA), Porto Alegre (RS), Brazil
João Quevedo National Science and Technology Institute (INCT) for Translational Medicine Laboratory of Neurosciences, Postgraduate Program in Health Sciences, Health Sciences Unit, Universidade do Extremo Sul Catarinense (UNESC), Criciúma (SC), Brazil

Nadja Schröder

National Science and Technology Institute (INCT) for Translational Medicine

Neurobiology and Developmental Biology Laboratory, School of Biosciences, Pontifícia Universidade Católica do Rio Grande do Sul (PUC-RS), Porto Alegre (RS), Brazil

José Alexandre S. Crippa

National Science and Technology Institute (INCT) for Translational Medicine Neurosciences and Behavior Department, Faculdade de Medicina de Ribeirão Preto (FMRP), Universidade de São Paulo (USP-RP), Ribeirão Preto (SP), Brazil 


\section{Disclosures}

\begin{tabular}{|c|c|c|c|c|c|c|c|}
\hline $\begin{array}{l}\text { Writting group } \\
\text { member }\end{array}$ & Employment & $\begin{array}{l}\text { Research } \\
\text { grant }^{1}\end{array}$ & $\begin{array}{c}\text { Other research grant } \\
\text { or medical continuous } \\
\text { education }\end{array}$ & $\begin{array}{l}\text { Speaker's } \\
\text { honoraria }\end{array}$ & $\begin{array}{c}\text { Ownership } \\
\text { interest }\end{array}$ & $\begin{array}{l}\text { Consultant } / \\
\text { Advisory } \\
\text { board }\end{array}$ & Other ${ }^{3}$ \\
\hline Flávio Kapczinski & UFRGS & $\begin{array}{c}\text { CNPq }^{*} \\
\text { CAPES }^{*} \\
\text { FIPE-HCPA } \\
\text { INCT-TM* }\end{array}$ & $\begin{array}{c}\text { Astra-Zeneca* } \\
\text { Stanley* } \\
\text { NARSAD* }^{*}\end{array}$ & $\begin{array}{c}\text { Lilly* }^{*} \\
\text { Astra-Zeneca* } \\
\text { Janssen* } \\
\text { Servier* }\end{array}$ & - & Servier* & - \\
\hline $\begin{array}{l}\text { Jaime Eduardo } \\
\text { Hallak }\end{array}$ & FMRP & $\begin{array}{l}\text { FAPESP }^{* *} \\
\text { CNPq }^{* *} \\
\text { CAPES }^{* *} \\
\text { FAEPA }^{*}\end{array}$ & FAEPA* & - & - & - & - \\
\hline $\begin{array}{l}\text { Antonio Egidio } \\
\text { Nardi }\end{array}$ & UFRJ & $\mathrm{CNPq}^{*}$ & $\begin{array}{c}\mathrm{CNPq}^{*} \\
\text { Eurofarma* }\end{array}$ & $\begin{array}{l}\text { Solvay* } \\
\text { Hoffmann } \\
\text { La-Roche* }\end{array}$ & - & $\begin{array}{l}\text { CNPq }^{*} \\
\text { Hoffmann La- } \\
\text { Roche }^{*}\end{array}$ & $\begin{array}{l}\text { Ed. Phoenix* } \\
\text { Segmento } \\
\text { Pharma* } \\
\text { Ed. Elsevier* } \\
\text { Eurofarma* }\end{array}$ \\
\hline Rafael Roesler & UFRGS & $\begin{array}{c}\text { CNPq } \\
\text { FIPE/HCPA } \\
\text { South } \\
\text { American } \\
\text { Officer for } \\
\text { Anticancer } \\
\text { Drug } \\
\text { Development } \\
\text { CAPES }\end{array}$ & - & - & $\begin{array}{c}\text { Co-founder } \\
\text { and partner } \\
\text { NeuroAssay } \\
\text { Research } \\
\text { and } \\
\text { Development } \\
\text { Ltd }\end{array}$ & $\begin{array}{c}\text { Eurofarma } \\
\text { Laboratórios } \\
\text { Instituto do } \\
\text { Câncer Infantil } \\
\text { do Rio Grande } \\
\text { do Sul } \\
\text { South American } \\
\text { Officer for } \\
\text { Anticancer Drug } \\
\text { Development } \\
\text { NeuroAssay } \\
\text { Research and } \\
\text { Development Ltd }\end{array}$ & - \\
\hline João Quevedo & UNESC & - & - & - & - & - & - \\
\hline Nadja Schröder & PUC-RS & $\begin{array}{l}\text { CNPq } \\
\text { CAPES }\end{array}$ & - & - & $\begin{array}{c}\text { Co-founder } \\
\text { and partner, } \\
\text { NeuroAssay } \\
\text { Research } \\
\text { and } \\
\text { Development } \\
\text { Ltd } \\
\end{array}$ & $\begin{array}{l}\text { Co-founder and } \\
\text { partner, } \\
\text { NeuroAssay } \\
\text { Research and } \\
\text { Development Ltd }\end{array}$ & - \\
\hline $\begin{array}{l}\text { José Alexandre S. } \\
\text { Crippa }\end{array}$ & FMRP & $\begin{array}{l}\text { FAPESP } \\
\text { CNPq }^{* *} \\
\text { CAPES }^{* *} \\
\text { FAEPA }^{*}\end{array}$ & FAEPA* & - & - & - & $\begin{array}{l}\text { FUNPEC }^{*} \\
\text { Pfiser }^{*} \\
\text { Elli-Lilly* }^{*} \\
\text { Astra- } \\
\text { Zeneca* }\end{array}$ \\
\hline \multicolumn{8}{|c|}{ 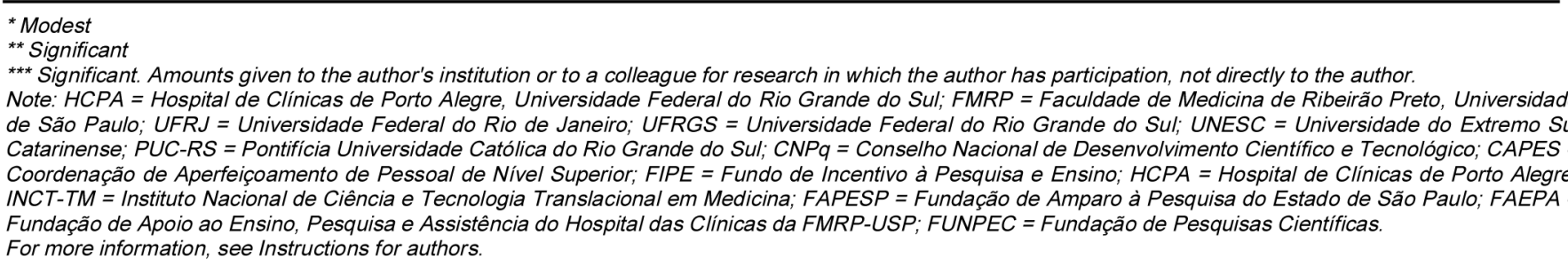 } \\
\hline
\end{tabular}

